\title{
Damping Coefficient Induces Stochastic Multiresonance in Bistable System with Asymmetric Dichotomous Noise
}

\author{
Juan $\mathrm{Wu}^{1,2}$ and Yong $\mathrm{Xu}^{2}$ \\ ${ }^{1}$ School of Mathematics \& Information Science, Beifang University for Nationalities, Yinchuan 750021, China \\ ${ }^{2}$ Department of Applied Mathematics, Northwestern Polytechnical University, Xian 710072, China \\ Correspondence should be addressed to Juan Wu; juanwuxixi@gmail.com
}

Received 2 June 2014; Revised 13 September 2014; Accepted 18 September 2014; Published 19 October 2014

Academic Editor: Gualberto Solís-Perales

Copyright (c) $2014 \mathrm{~J}$. Wu and Y. Xu. This is an open access article distributed under the Creative Commons Attribution License, which permits unrestricted use, distribution, and reproduction in any medium, provided the original work is properly cited.

\begin{abstract}
Stochastic resonance (SR) and stochastic multiresonance (SMR) phenomena as a function of the underdamping and overdamping coefficients in bistable system with asymmetric dichotomous noise are investigated numerically. By the efficient numerical simulation of the asymmetric dichotomous noise and the fourth-order Runge-Kutta algorithm, we calculate the system responses, the averaged power spectrum, and the signal-noise-ratio (SNR) that can be a measure of the existence of SR and SMR phenomenon. And the effects of damping coefficients on the three characteristics are analyzed. Firstly, it is found that the periodic asymmetric distribution of the particle's hopping between two potential wells in the system response is gradually weakened as underdamping coefficient is increased to overdamping coefficient. And it also displays the periodic asymmetric distribution under the circumstance of overdamping coefficient. Then the averaged power spectrum exhibits multiple sharp peaks, and the highest peak increases and decreases for underdamping coefficient which is added to overdamping coefficient. Finally, SNR versus the damping coefficient for the system parameters and the noise parameters are acquired and they show multiple peaks and valleys, which illustrates the obvious SMR phenomena in bistable system with asymmetric dichotomous noise.
\end{abstract}

\section{Introduction}

Previously, noise is regarded as an ingredient that has a negative effect on system. Nevertheless the appearance of SR phenomenon alters people's attitude, which assists the sequence of the disordered system to be well organized. Given this, SR has been investigated profoundly by a number of scientists. SR phenomenon was firstly proposed to explain the periodicity in the earth's ice ages [1]. Then SR phenomenon was observed in various fields in nature, for instance, physics, chemistry, biology, ecology, graphics, and so on [2]. Up to now, there are abundant publications on SR based on the original discovery of SR, and the conception of SR was developed in a broad sense, for example, SMR, quantum SR, autonomous SR, aperiodic SR, coherence resonance, and logical SR [3-16].

SR and SMR are thought to be a kind of the coherent manner among nonlinear system, random noise, and periodic force. And various characteristics can be used to measure the emerging of SR and SMR phenomena, such as the amplitude of system response, the output amplitude gain, the averaged power spectrum, the spectral power amplification, the signal-noise-ratio, the residence time distributions, and the information and probability of detection, by which SR and SMR phenomena can be easily explored [2]. For example, Ray and Sengupta measured SR in underdamped bistable system by the power spectrum [17], Xu et al. found SR phenomena in a bistable system with Lévy noise by the SNR [18], and Zhang et al. demonstrated SMR phenomena in a linear system driven by multiplicative polynomial dichotomous noise by the SNR [19]. In this paper, the amplitude of system response, the averaged power spectrum, and the SNR are the main characteristic indicators to demonstrate the existence of SR and SMR phenomena.

As we know, SR and SMR phenomena in overdamped bistable system and underdamped bistable system have been researched widely both theoretically and experimentally for their applications [17, 20-23]. However, fewer investigators pay attention to SR and SMR phenomena in the overdamped and underdamped bistable system simultaneously. Thus, we 
will focus on SR and SMR phenomena versus the damping coefficients. Besides, a mass of the researches focus on Gaussian noise or white noise just for its simplicity. But they are just a sort of ideal model of the actual noises, and they cannot represent noise with the exponential relevance. Nevertheless asymmetric dichotomous noise is a non-Gaussian color noise and is widespread for its manageability in some fields, which leaps between two fixed points $(a,-b,(a, b>0))$; its waiting time is submitted to the exponential distribution, and this kind of the leaps inspires the nonequilibrium more easily than Gaussian noise [24]. In addition, up to now SR and SMR phenomena about the dichotomous noise have been studied largely in theory. Jin, $\mathrm{Li}$, and some other experts have done some great work theoretically on SR in typical systems with the dichotomous noise [25-27]. And the research of the numerical simulation of SR and SMR phenomena is less relative. Consequently in this paper we will explore SR and SMR phenomena versus the damping coefficients in bistable system with asymmetric dichotomous noise numerically.

This paper is organized as the following. In Section 2 , the bistable system and asymmetric dichotomous noise are introduced. In Section 3, SR and SMR phenomena are researched in three aspects. In Section 3.1, SR phenomenon is investigated by the transition between the two potential wells of the response of the system driven by the asymmetric dichotomous noise. In Section 3.2, the averaged power spectrum is computed numerically, and the signature of SR phenomenon is obviously displayed. In Section 3.3, we obtain the SNR versus the damping coefficient. And there are multiple peaks in the SNR which demonstrates the existence of SMR phenomena in the bistable system with asymmetric dichotomous noise.

\section{Bistable System with Asymmetric Dichotomous Noise}

We consider a bistable system with a periodic signal driven by asymmetric dichotomous noise, which can be described by the following Langevin equation:

$$
\frac{d^{2} x}{d t^{2}}=-\eta \frac{d x}{d t}-\frac{d V(x)}{d x}+F(t)+\varphi(t)
$$

where $\eta$ is the damping coefficient. It is an underdamped bistable system when $\eta<1$; it is an overdamped bistable system when $\eta>1 . V(x)$ is the double potential well function defined as $-m x^{2} / 2+n x^{4} / 4(m>0, n>0)$, which has two stable points at $V_{ \pm}= \pm \sqrt{\mathrm{m} / n}$, and the height of potential barrier is $\Delta V(x)=m^{2} / 4 n$. When we choose the parameters as $m=n=1$, it is the standard double potential well function. And the two stable fixed points are $V_{ \pm}= \pm 1, \Delta V(x)=$ $1 / 4 . F(t)$ is the periodic signal, which can be described as $A \cos (\omega t+\theta)$, and $A$ and $\omega$, respectively, are the amplitude and forcing frequency of the periodic signal.

$\varphi(t)$ is asymmetric dichotomous noise which jumps between two values $a$ and $b$ with mean waiting times $t_{a}$ and $t_{b}$, and the rates of the switching can be obtained from the mean waiting; that is $\mu_{a}=1 / t_{a}, \mu_{b}=1 / t_{b}$.
The master equation of this noise can be described as

$$
\begin{aligned}
& \partial_{t} P\left(a, t \mid x, t_{0}\right)=-\mu_{a} P\left(a, t \mid x, t_{0}\right)+\mu_{b} P\left(b, t \mid x, t_{0}\right), \\
& \partial_{t} P\left(b, t \mid x, t_{0}\right)=\mu_{a} P\left(a, t \mid x, t_{0}\right)-\mu_{b} P\left(b, t \mid x, t_{0}\right),
\end{aligned}
$$

with the initial condition and the total probability condition:

$$
\begin{gathered}
P\left(x^{\prime}, t \mid x, t_{0}\right)=\delta_{x^{\prime} x}, \\
P\left(a, t \mid x, t_{0}\right)+P\left(b, t \mid x, t_{0}\right)=1 .
\end{gathered}
$$

Then the solution of the master equation is

$$
\begin{aligned}
P\left(a, t \mid x, t_{0}\right)= & \frac{\mu_{b}}{\mu_{b}+\mu_{a}}+\left(\frac{\mu_{a}}{\mu_{b}+\mu_{a}} \delta_{a x}-\frac{\mu_{b}}{\mu_{b}+\mu_{a}} \delta_{b x}\right) \\
& \times \exp \left(-\left(\mu_{a}+\mu_{b}\right)\left(t-t_{0}\right)\right), \\
P\left(b, t \mid x, t_{0}\right)= & \frac{\mu_{a}}{\mu_{b}+\mu_{a}}-\left(\frac{\mu_{a}}{\mu_{b}+\mu_{a}} \delta_{a x}-\frac{\mu_{b}}{\mu_{b}+\mu_{a}} \delta_{b x}\right) \\
& \times \exp \left(-\left(\mu_{a}+\mu_{b}\right)\left(t-t_{0}\right)\right) .
\end{aligned}
$$

And the stationary solution of (2) can be easily obtained as

$$
\begin{aligned}
& P_{s}(a)=\frac{\mu_{b}}{\mu_{a}+\mu_{b}}, \\
& P_{s}(b)=\frac{\mu_{a}}{\mu_{a}+\mu_{b}} .
\end{aligned}
$$

Then the stationary mean which can be obtained by using (4) is

$$
\langle\xi(t)\rangle_{s}=\frac{a \mu_{b}+b \mu_{a}}{\mu_{a}+\mu_{b}},
$$

and the stationary correlation function is

$$
\begin{aligned}
\left\langle\xi(t) \xi\left(t^{\prime}\right)\right\rangle= & \left(\frac{b \mu_{a}+a \mu_{b}}{\mu_{b}+\mu_{a}}\right)^{2}+\frac{\mu_{a} \mu_{b}(a-b)^{2}}{\left(\mu_{b}+\mu_{a}\right)^{2}} \\
& \times \exp \left(-\left(\mu_{a}+\mu_{b}\right)\left(t-t^{\prime}\right)\right) .
\end{aligned}
$$

Here the mean function and the correlation function satisfy the following conditions:

$$
\begin{gathered}
\langle\xi(t)\rangle=0, \\
\left\langle\xi(t) \xi\left(t^{\prime}\right)\right\rangle=\lambda D \exp \left(-\lambda\left|t-t^{\prime}\right|\right),
\end{gathered}
$$

where $D$ is the noise intensity and $\lambda$ is the noise correlation time. Thus, the noise intensity of the asymmetric dichotomous noise can be computed as

$$
D=\frac{1}{2} \int_{-\infty}^{\infty}\left(\langle\xi(\tau) \xi(0)\rangle-\langle\xi(\tau)\rangle^{2}\right) d \tau=\frac{(a-b)^{2} \mu_{a} \mu_{b}}{\left(\mu_{a}+\mu_{b}\right)^{3}} .
$$

Finally, the rates of the switching $\mu_{a}$ and $\mu_{b}$ of the asymmetric dichotomous noise are computed in terms of the state values 


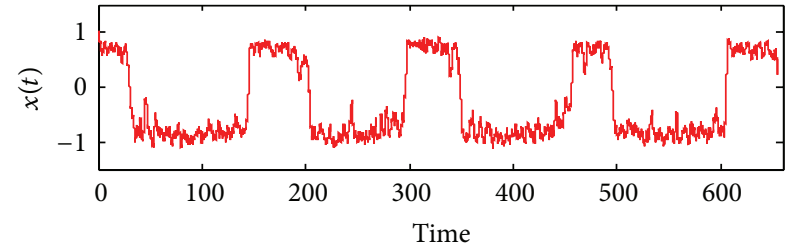

(a)

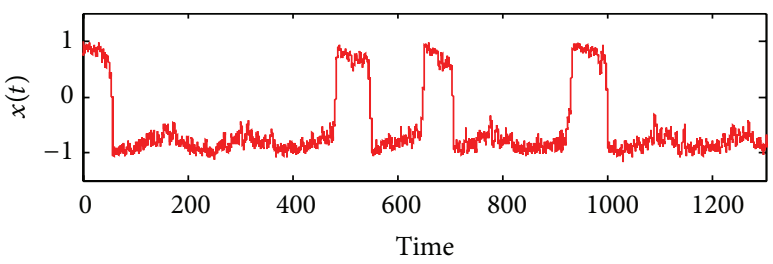

(c)

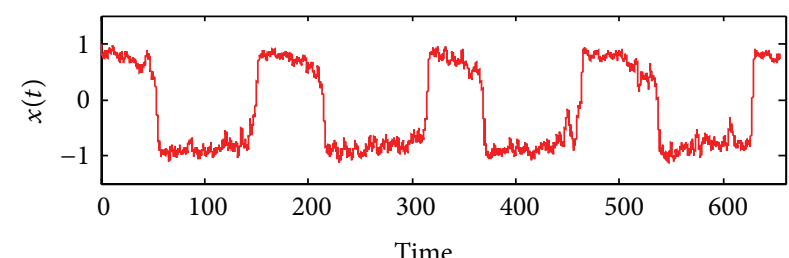

(b)

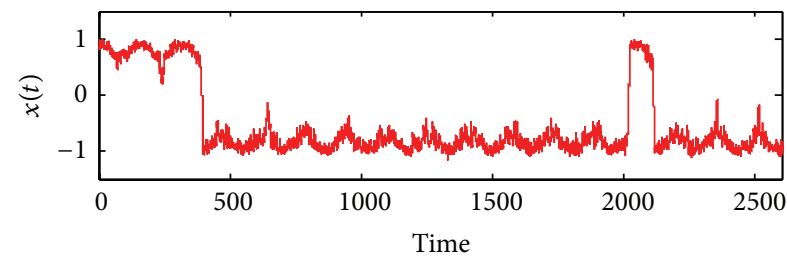

(d)

FIGURE 1: The system responses of the underdamped and overdamped bistable system (a) $\eta=0.2$, (b) $\eta=0.6$, (c) $\eta=1.0$, and (d) $\eta=1.4$.

and the noise intensity. Also the conditional probabilities $P_{a a}$ and $P_{b a}$ can also be computed as follows:

$$
\begin{aligned}
P_{a a}=P\left(a, t_{n+1} \mid a, t_{n}\right)= & \frac{\mu_{b}}{\mu_{b}+\mu_{a}}+\frac{\mu_{a}}{\mu_{b}+\mu_{a}} \\
& \times \exp \left(-\left(\mu_{a}+\mu_{b}\right) d t\right), \\
P_{b a}=P\left(a, t_{n+1} \mid b, t_{n}\right)= & \frac{\mu_{b}}{\mu_{b}+\mu_{a}}-\frac{\mu_{b}}{\mu_{b}+\mu_{a}} \\
& \times \exp \left(-\left(\mu_{a}+\mu_{b}\right) d t\right) .
\end{aligned}
$$

And the numerical series of the asymmetric dichotomous noise can be obtained by the above formulas and the following relevant algorithm. The algorithm procedure of the asymmetric dichotomous noise can be described as follows. Firstly, the initial state of the asymmetric dichotomous noise can be supposed to be $x_{0}=a,\left(x_{0}=b\right)$; a series of random numbers $R_{n}(n=0,1,2, \ldots)$ in the interval $[0,1]$ are generated in computer, which are compared with the conditional probability $P_{a a}$ or $P_{b a}$. Then we consider that if $R_{0}<P_{a a}\left(R_{0}<P_{b a}\right)$, we will ascertain $x_{1}=a$, or else $x_{1}=b$. Next, we also should decide that if $R_{1}<P_{a a}\left(R_{1}<P_{b a}\right)$, we will ascertain $x_{2}=a$, or else $x_{2}=b$. And keep doing this. Finally, a series of random numbers $R_{n}$ are got in computer. Moreover, the timestep $d t$ should be much lesser [28].

\section{Stochastic Multiresonance}

We are devoted to researching SR and SMR phenomena of this bistable system induced by the change of the damping coefficients in this section. According to this complex twodimensional system, numerical simulation method is a good research approach, so the specific numerical simulation program is shown in regard to SR and SMR in bistable system with asymmetric dichotomous noise. And we study the system responses, the averaged power spectrum, and the SNR which can be used to reflect SR phenomenon.

For the system responses, the particle oscillates at the bottom of the one potential well at a level of the certain damping coefficients, and then it oscillates between the two potential wells with the increasing and decreasing of the damping coefficients. That is to say, SR phenomenon can be discovered with the damping coefficients of the system. And the averaged power spectrum displays the relationship between the system, the cosine signal, and the asymmetric dichotomous noise for different underdamping and overdamping coefficients, from which we can find SR phenomenon. Moreover, we also devote ourselves to computing the SNR as one important symbol of SR phenomenon. If the SNR gives rise to one or more extreme values as we modulate the damping coefficients from the underdamping to overdamping at a certain range of parameters, it shows that the bistable system with asymmetric dichotomous noise has presented SR phenomenon or SMR phenomena.

3.1. System Responses. In order to obtain the responses of this system, the bistable system is transformed into two onedimensional systems as

$$
\begin{aligned}
& \frac{d x}{d t}=y, \\
& \frac{d y}{d t}=-\eta y-\frac{d V(x)}{d x}+F(t)+\varphi(t),
\end{aligned}
$$

and then the system responses are calculated by the discretization of the above form and the fourth-order RungeKutta algorithm.

We fix the parameters of the system, the signal, and the asymmetric dichotomous noise as $A=0.2, \theta=0.03, \omega=$ $0.04, D=0.01, a=3.0, b=-1.0$. And the effects of the different damping coefficients including the underdamping coefficients and overdamping coefficients on the SNR are investigated. In Figure 1(a), the underdamping coefficients of this system is 0.2 , and we can find that the particle oscillates in the $V_{+}=1$ around, surmounts the potential barrier, jumps into and oscillates around the $V_{-}=-1$. Then the particle oscillates in the $V_{-}=-1$ around, surmounts the potential barrier, and oppositely jumps into and oscillates around the 


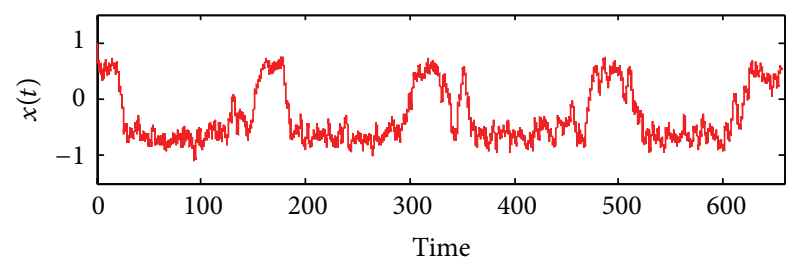

(a)

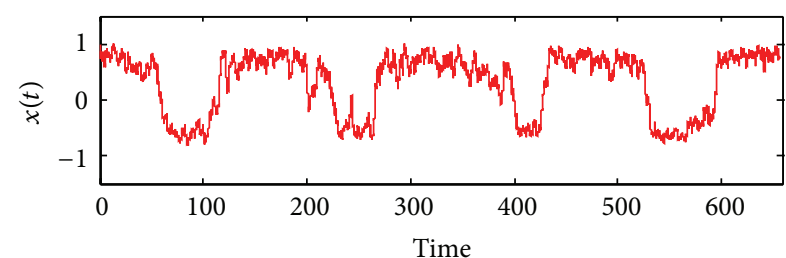

(b)

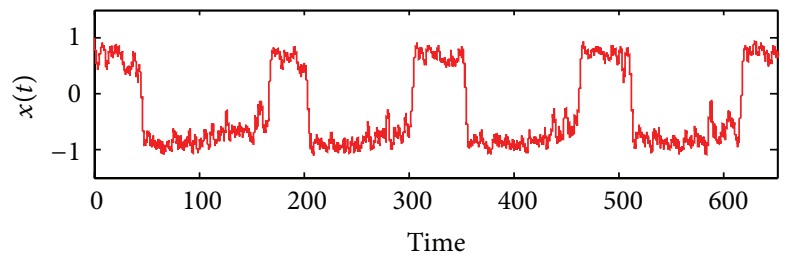

(c)

FIGURE 2: The system responses of the overdamped bistable system for different states values of the asymmetric dichotomous noise (a) $a=$ $4.0, b=-2.0$, (b) $a=2.0, b=-4.0$, and (c) $a=3.0, b=-1.0$.

$V_{+}=1$. For some time, the periodic transition of the particle between the two potential wells and the uniform asymmetry are obviously shown. With the increasing of the underdamping coefficients to 0.6, Figure 1(b) also clearly reflects the periodic transition of the particle between the two potential wells and the uniform asymmetry. When the underdamping coefficients of this system is increased to 1.0, the underdamped bistable system transforms into the overdamped one, the transition of the particle between the two potential wells and the asymmetry of the distribution can also be found but the clear periodicity is not occurred, and it is important that the particle remains mostly in the $V_{-}=-1$. Finally, when the overdamping coefficients are continually increased to 1.4, compared with the case of Figure 1(c), the transition between the two potential wells still remains, and the most important thing is that the asymmetry of the distribution is more severe and the vast majority of the states are located in the $V_{-}=-1$, yet the initial state is located in the $V_{+}=1$ in Figure 1(d).

According to three subfigures of Figure 2, we pay attention to the overdamped bistable system and the asymmetry of the system response. In Figure 2(a), the overdamping coefficient is adjusted to 1.3 , and the asymmetric dichotomous noise parameter is increased to $a=4.0, b=-2.0$. It is illustrated that the particle oscillates between the two potential wells, the intense asymmetry of the distribution exists, and most states are located in the $V_{-}=-1$; meanwhile the random vibration is intense compared with Figure 1(d). In Figure 2(b), we interchange the state value of the asymmetric dichotomous noise $a=2.0, b=-4.0$; it is clear that the asymmetry of the distribution and the action of the random vibration still exist; nevertheless most states are located in the $V_{+}=1$ conversely, compared with Figure 2(a). Moreover, with the asymmetric dichotomous noise parameter decreased to $a=3.0, b=-1.0$, Figure 2(c) shows us the more regular asymmetry of the distribution as the action of the random vibration is weak.

3.2. Averaged Power Spectrum. Power spectrum can reflect a kind of the coordination between the signal and the noise. But it includes a large number of random factors. So we make use of the method of average to eliminate the random factors and then obtain the averaged power spectrum which reflects the coefficient properties of the cosine signal and the asymmetric dichotomous noise. Accordingly, whether the damping coefficient including the underdamping and the overdamping can induce SR phenomenon in terms of the averaged power spectrum is the cure of our research in this section.

The power spectrum density can be obtained by the following formula of the Fourier transform of the autocorrelation function:

$$
P(\omega)=\int_{-\infty}^{\infty}\langle x(t+\tau) x(t)\rangle \exp (-i \omega \tau) d \tau .
$$

Next, it is found that ensemble averaging on 600 power spectrum trajectories is more sufficient to achieve the averaged power spectrum. Thus several averaged power spectrum figures are obtained as the following for the different damping coefficient.

In Figure 3(a), we choose the damping coefficient $\eta=$ 0.05 , which means that the system becomes the underdamped bistable system with the fixed parameters $A=1.0, a=$ $3.0, b=-1.0, D=0.001, \omega=1.0, \theta=0.03$. And it is easily observed that three distinct peaks appear on the averaged power spectrum due to the effect of the periodicity of the periodic signal and the asymmetry of the asymmetric dichotomous noise on the system, although the underdamping coefficient is smaller. They are marked $P_{1}, P_{2}$, and $P_{3}$ from left to right so as to reveal the meaning that the figure contains conveniently and particularly. Between the three peaks, the value of the middle peak $P_{2}$ is the highest; it is almost double $P_{3}$ 's, and the value of the left peak $P_{1}$ is the lowest. Then the underdamping coefficient is added to 0.1 as other parameters are invariant, and three distinct peaks are still observed in Figure 3(b). Here the value of the highest peak $P_{2}$ is rapidly increased, but the value of $P_{3}$ is decreased and the value of $P_{1}$ is decreased slightly. And as it is increased to $0.3,0.6,0.9,1.0$, and 1.3 , the similar increase and decrease are clearly present in Figures 3(c), 3(d), 3(e), 3(f), and 3(g). 


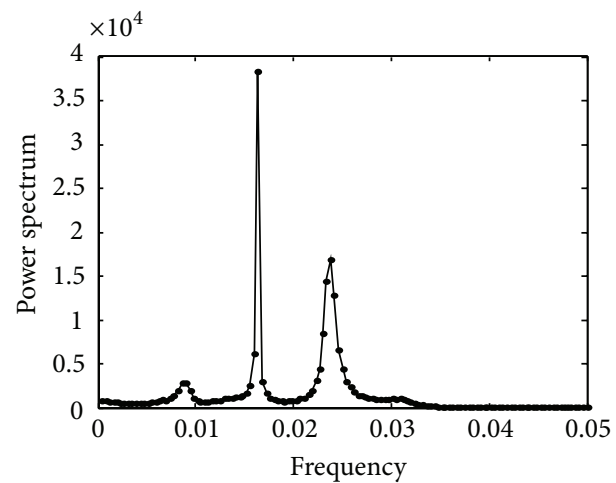

(a)

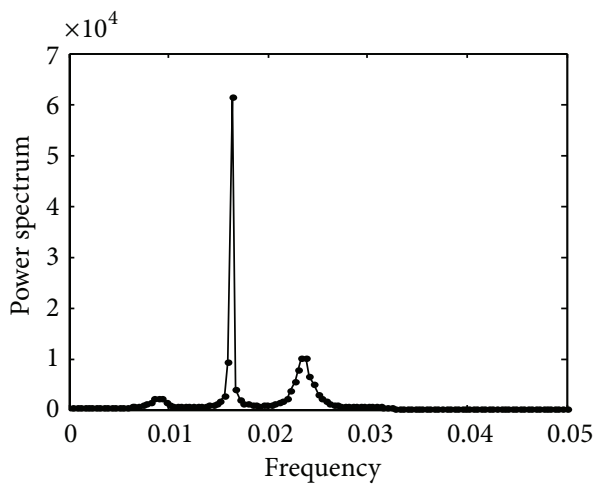

(b)

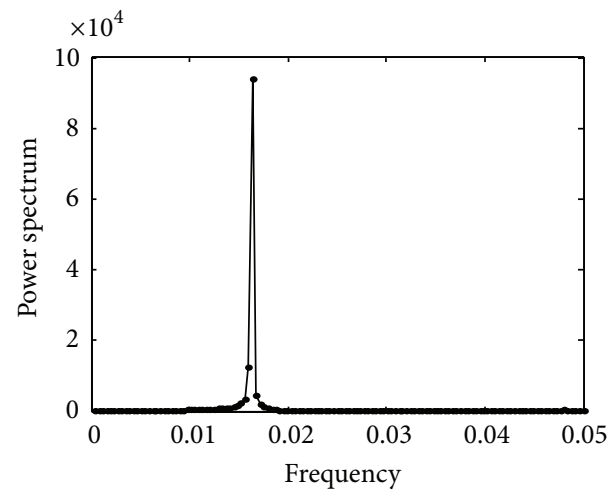

(d)

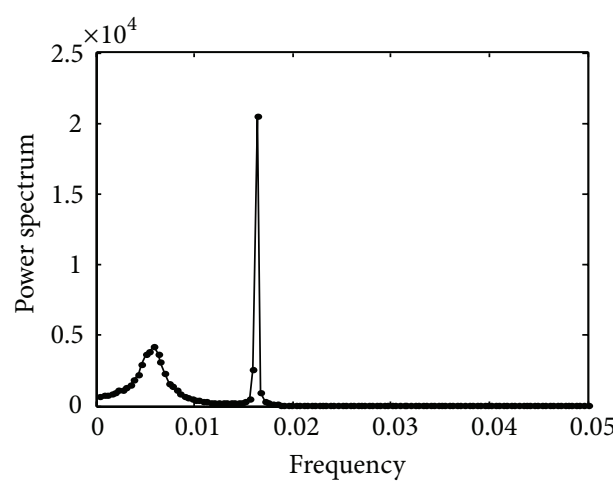

(f)

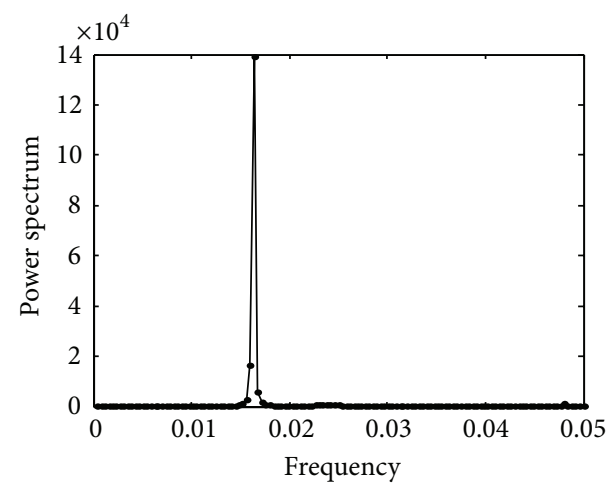

(c)

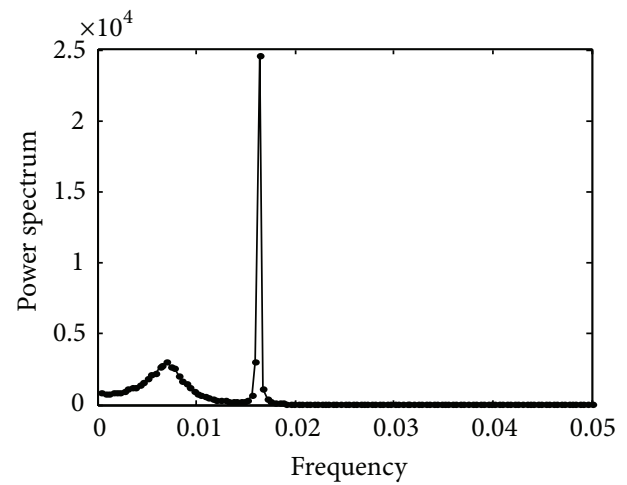

(e)

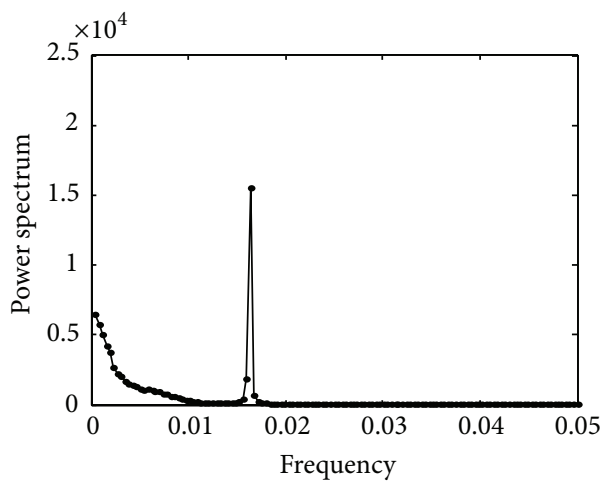

(g)

FIGURE 3: The averaged power spectrum of the system for different damping coefficient (a) $\eta=0.05$, (b) $\eta=0.1,(\mathrm{c}) \eta=0.3$, (d) $\eta=0.6$, (e) $\eta=0.9$, (f) $\eta=1.0$, and (g) $\eta=1.3$. 
In general, when the damping coefficient is increased gradually from the underdamping to overdamping, the value of $P_{2}$ that is the highest peak increases sharply and then decreases slowly, the value of $P_{3}$ that is the second highest peak decreases all the time, and the value of $P_{1}$ that is the lowest peak decreases firstly and then increases slowly and disappears last. And all those variations of the three peaks with the change of damping coefficient reveal SR phenomena.

3.3. Signal-Noise-Ratio. SNR is a typical method to measure SR and SMR phenomena. And in this section as a function of damping coefficient it displays the conspicuous SR and SMR phenomena. At present, there are many several numerical simulation methods about SNR. We employ the following formula [29]:

$$
\mathrm{SNR}=10 \log \frac{P_{S}(\omega)}{P_{N}(\omega)},
$$

where $P_{S}(\omega)$ and $P_{N}(\omega)$ are the output power spectrum of the periodic signal and the asymmetric dichotomous noise, respectively. And in the following figures, the peaks of the SNR phenomenon are marked as $S_{1}, S_{2}, S_{3}$, and $S_{4}$ similarly in order to represent those figures conveniently and particularly.

In Figure 4, SNR as the functions of the damping coefficient for the different amplitudes of signal and the fixed system $A=1.0,0.7,0.4, a=3.0, b=-1.0, D=0.001$, $\omega=1.0, \theta=0.03$, displays the conspicuous SR and SMR phenomena. It is clearly showed that, as the damping coefficient increases from 0.04 to 1.4, the SNR firstly increases sharply, next decreases slowly, and then increases more slowly. The nonmonotonic behaviors of the SNR obviously reveal the occurrence of SMR phenomenon. At the same time, the value of the peak descends slowly and then rapidly; also the value of the valley descends always and it moves towards the left, when the amplitude of the signal decreases from 1.0 to 0.7 and then to 0.4 .

In Figure 5, we observe the SNR versus the damping coefficient for the different forcing frequency of the periodic signal, and four subfigures represent the changes of SNR with the fixed system parameter $A=1.0, a=3.0, b=-1.0, D=$ $0.001, \theta=0.03$. In Figure 5(a), the obvious SR phenomenon is easily observed, but SR phenomenon demonstrates to us that there are two peaks; in other words, it is SMR phenomenon. And as the forcing frequency of the periodic signal $\omega$ is reduced from 1.5 to 1.4 and to 1.3 , the peaks $S_{1}$ and $S_{2}$ heighten and move right gradually and they become more and more evident, while it barely heighten when $\omega$ is reduced from 1.4 to 1.3 according to $S_{2}$. And when it is decreased from 1.3 to 0.7 , the similar move, increase, and decrease of the peaks are present, respectively, in Figures 5(b), 5(c), and 5(d).

In short, as the forcing frequency of the periodic signal constantly lessens from 1.5 to 0.7 , the SNR demonstrate the variation between two peaks and one peak, which can declare that the noteworthy SMR phenomena exist in the bistable system with asymmetric dichotomous noise.

In Figure 6, similarly we focus on the SNR versus the damping coefficient for the different state $a$ of the asymmetric dichotomous noise with the fixed system parameters $A=1.0$,

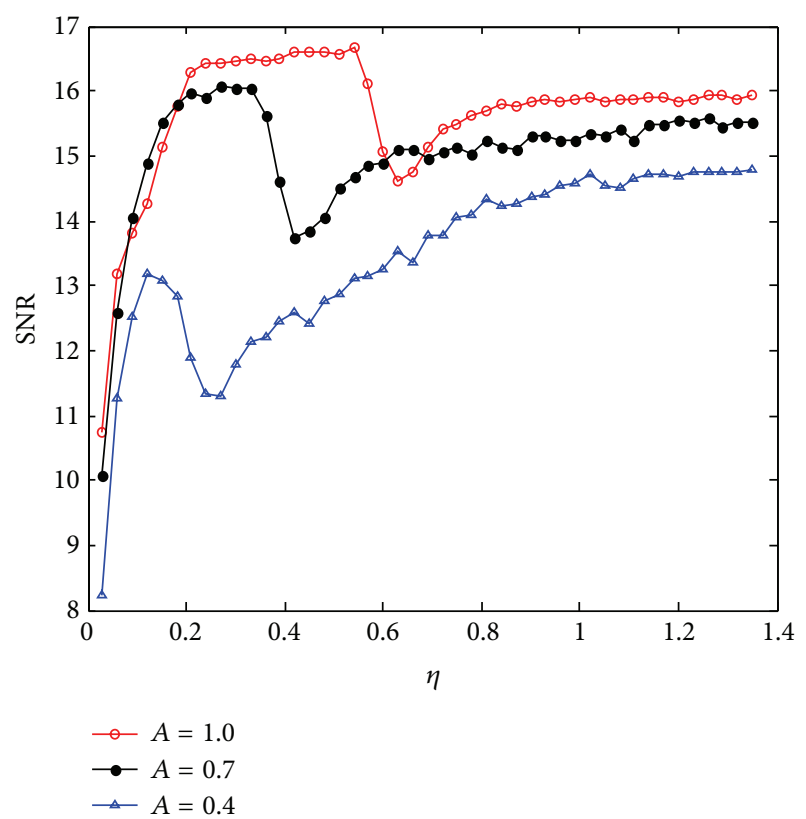

FIgURE 4: SNR versus the damping coefficient for the different amplitudes of the signal.

$\theta=0.03, \omega=1.3, b=-1.0, D=0.001$. According to the states of the asymmetric dichotomous noise, the state $b$ is fastened and the state $a$ is changed continually. When the state $a$ is selected as $a=4.0$ and the other state is $b=-1.0$, the noise is still the asymmetric dichotomous noise. It can be easily found that there are two peaks in the SNR, which go up increasingly but do not move toward left and right, when the state $a$ is reduced to 3.0 and then to 2.0. However, three distinct peaks turn up as the state $a$ is changed to 0.5 . During the process of the decrease of the state $a$, the SNR show us two peaks and three peaks, which demonstrates SMR phenomena.

The effect of the different asymmetric states of dichotomous noise on the SNR versus the damping coefficient is just investigated for SMR phenomena. Now we research how the symmetric states of dichotomous noise influence SMR phenomena versus the damping coefficient. And the parameter of the periodic signal and the noise is fastened $A=1.0, \theta=0.03, \omega=1.3, D=0.001$. By the clear manifestation of Figure 7, it is found that the SNR versus the damping coefficient present multiple peaks with the different symmetric states of dichotomous noise. When it is $a=$ $3.0 b=-3.0$, there are a peak and a valley. As the states are lessened to $a=2.0 b=-2.0$, the peak and the valley rise up but their positions do not shift to the left or the right; meanwhile another peak appears in the SNR. When the states are continuously reduced to $a=1.0 b=-1.0$, the peak $S_{1}$ and the valley go up and similarly their positions do not shift left and right; the peak $S_{2}$ yet develops into two peaks. And then the three peaks $S_{1}, S_{2}$, and $S_{3}$ get more remarkable, when the states are lessened lower $a=0.5 b=-0.5$. So SMR phenomena are always present, when the state of the symmetric dichotomous noise is increased constantly. 


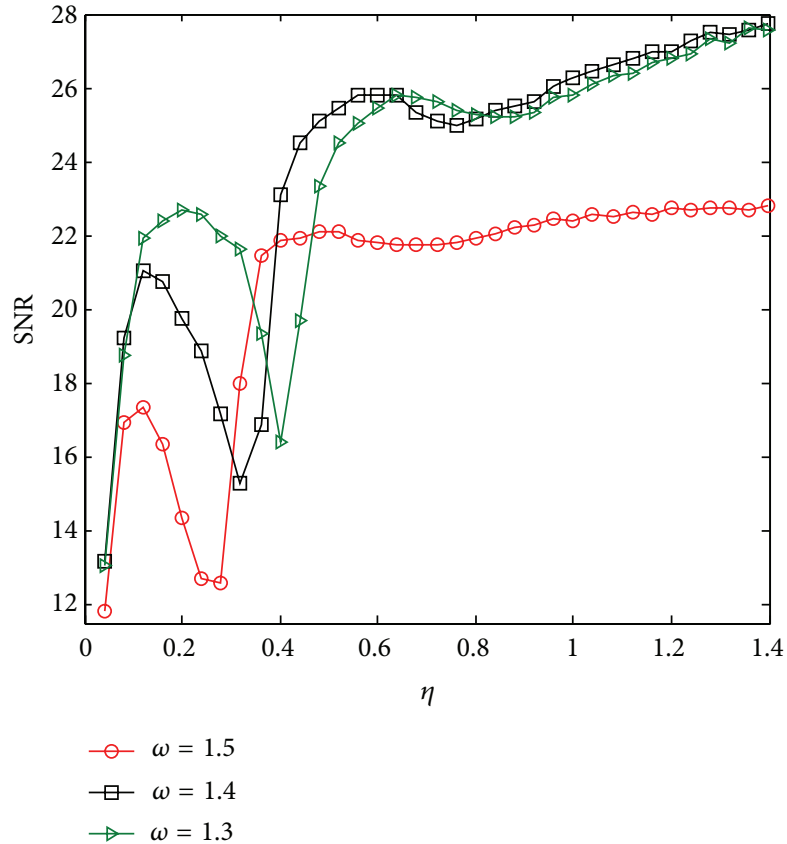

(a)

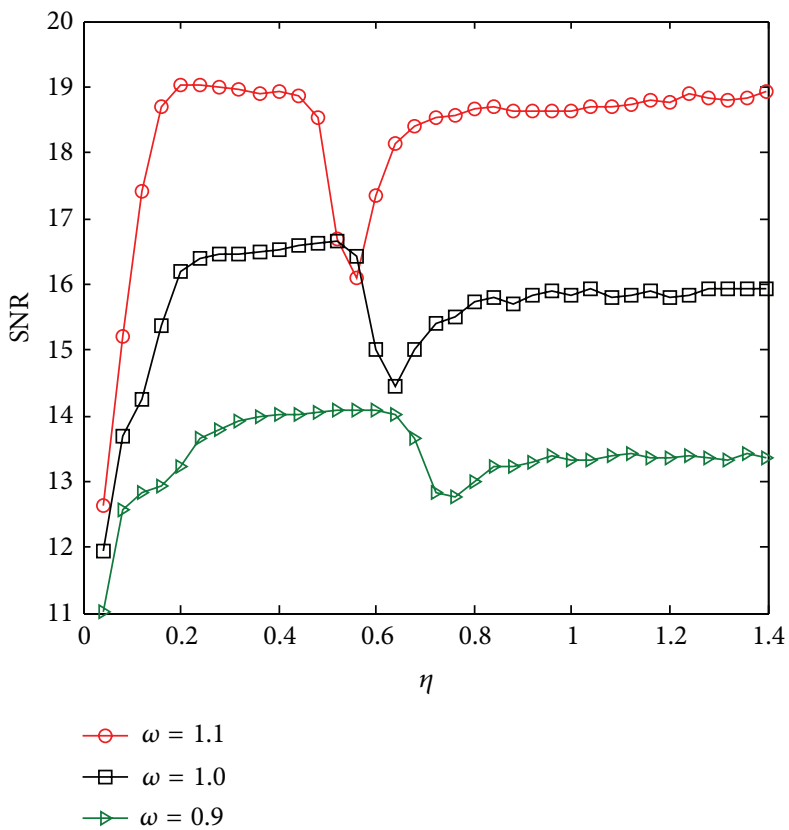

(c)

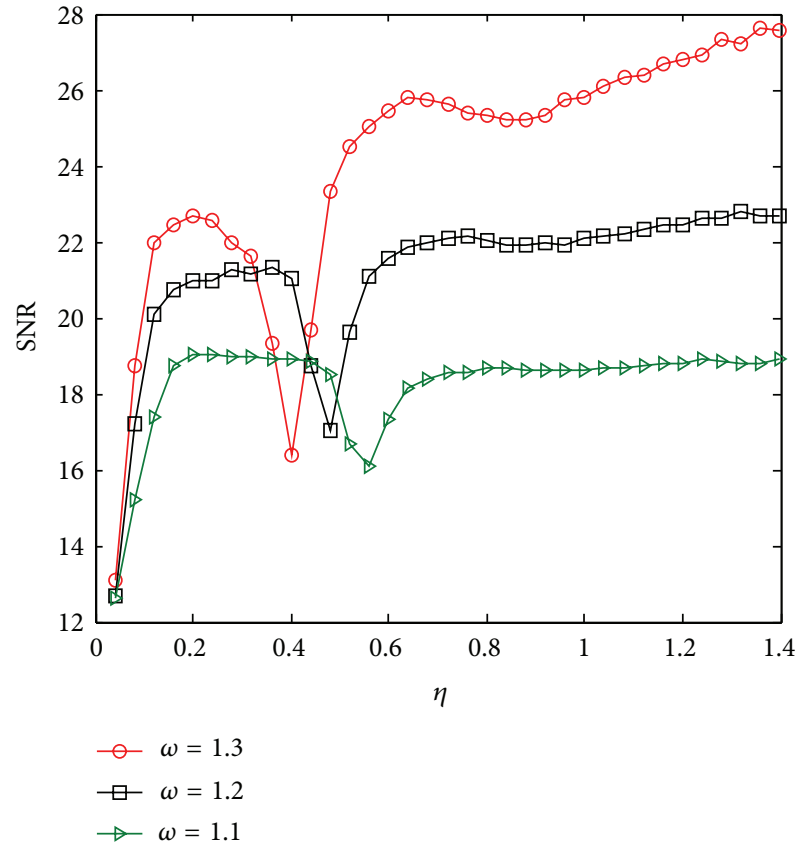

(b)

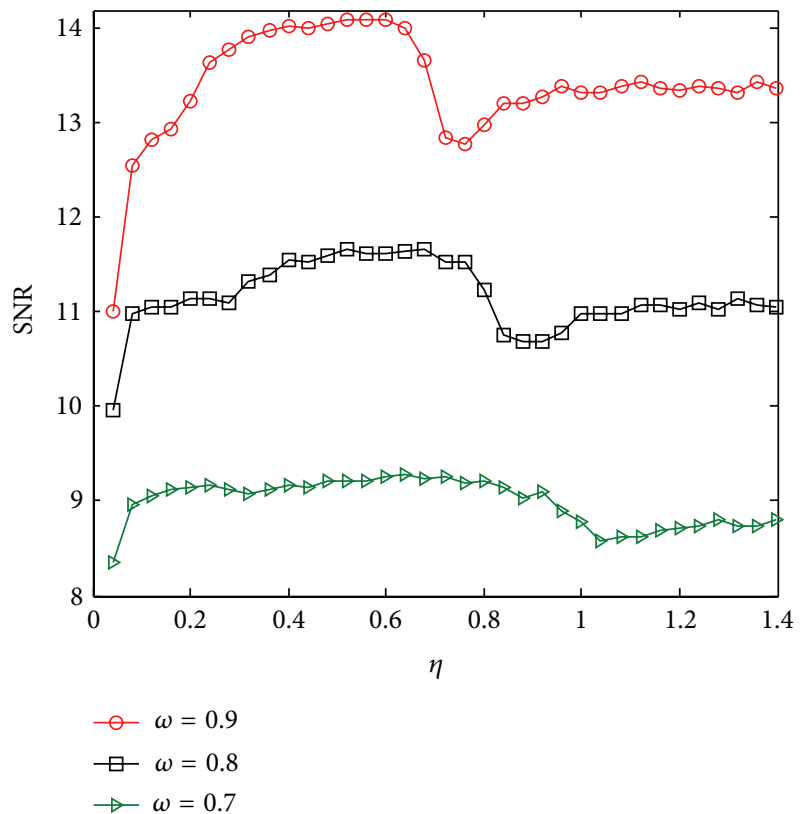

(d)

FIGURE 5: SNR versus the damping coefficient for the different forcing frequencies of the periodic signal.

Finally, whether the noise intensity of the asymmetric dichotomous noise can cause the prominent influence on the peak of the SNR is researched. Figure 8 shows us how the SNR changes based on the fixed parameters $A=1.0, \theta=0.03$, $\omega=1.3, a=0.5, b=-1.0$ with the increase of the noise intensity. It is demonstrated that three noteworthy peaks $S_{1}$, $S_{2}$, and $S_{3}$ appear in the SNR, when the noise intensity is 0.001 . And as it is increased to 0.01 , the peak $S_{2}$ disappears, and the peaks $S_{1}, S_{3}$ descend. There are two peaks in the SNR, which are marked again as $S_{1}, S_{2}$. Then we continue to improve the noise intensity to 0.2 and find that the two peaks $S_{1}$ and $S_{2}$ both decline gradually. Until the noise intensity is increased to 0.3 , the peaks $S_{1}$ and $S_{2}$ decline gradually once again, but it is particular that the peak $S_{2}$ disappears. There are a peak and a valley in the SNR with $D=0.03$. And all give evidence of the existence of SMR phenomena.

Furthermore, some situations in Figures 6, 7, and 8 are different from Figures 4 and 5 's, which demonstrate to us that 


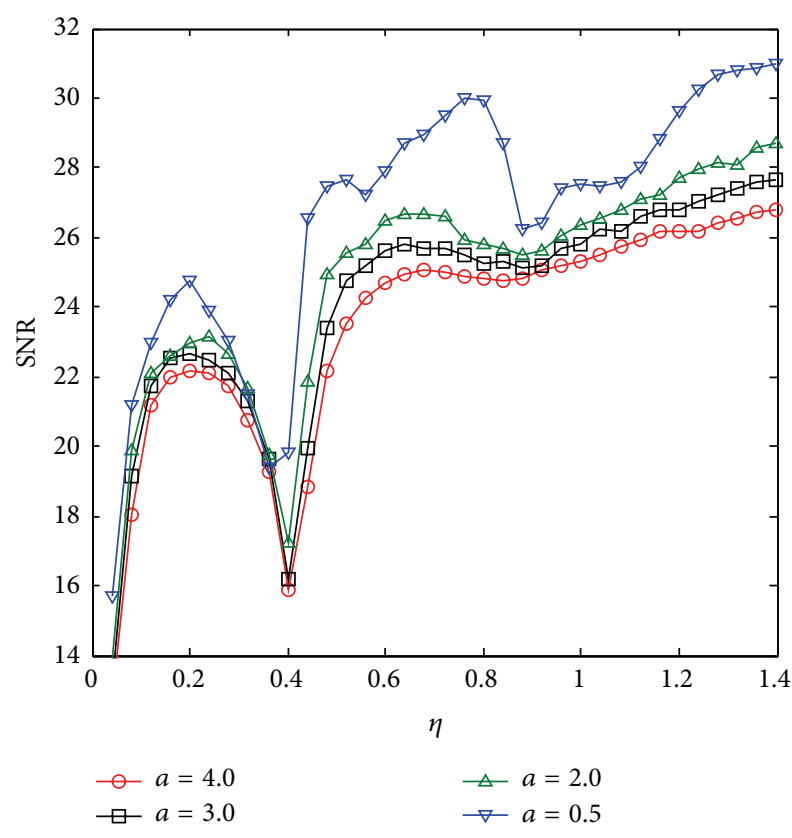

FIGURE 6: SNR versus the damping coefficient for the different state values of the asymmetric dichotomous noise.

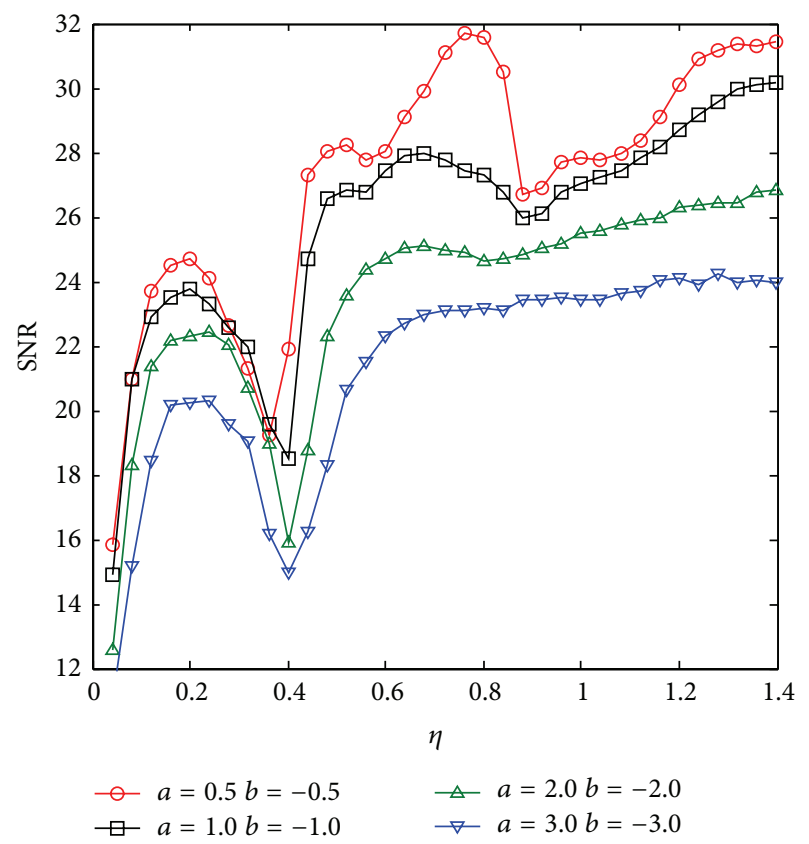

FIGURE 7: SNR versus the damping coefficient for the different state values of the symmetric dichotomous noise.

the amplitude and the forcing frequency of the periodic signal can change the position toward left and right of the peaks of SNR versus the damping coefficient, while the states and the intensity of the asymmetric dichotomous noise do not change the position of the peaks toward left or right but the high of the peaks.

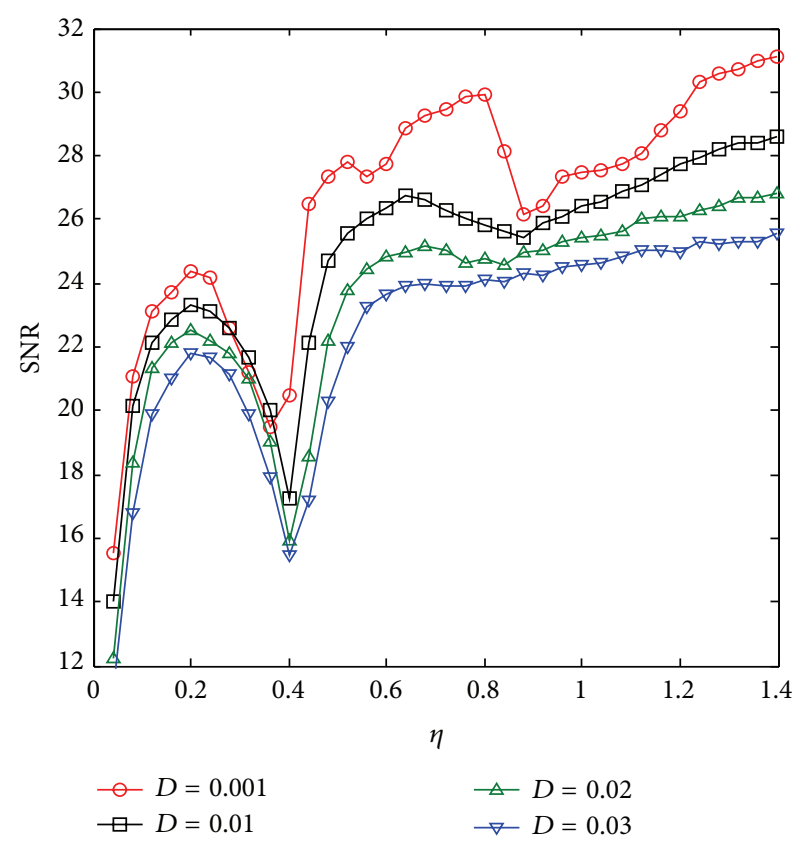

FIGURE 8: SNR versus the damping coefficient with the different noise intensity of the asymmetric dichotomous noise.

\section{Discussion and Conclusion}

Stochastic resonance (SR) and stochastic multiresonance (SMR) phenomena versus the damping coefficient in bistable system with asymmetric dichotomous noise have been researched numerically in this paper. The system response, the averaged power spectrum, and the signal-noise-ratio (SNR) have been applied to investigate and demonstrate SR and SMR phenomena.

Firstly, by the fourth-order Runge-Kutta numerical algorithm, it is found that the asymmetric dichotomous noise can induce the uniform asymmetry and the irregular asymmetry of the system response in the bistable system with the appropriately fixed parameters, as the damping coefficient is increased gradually from the underdamping 0.2 to the overdamping 1.4. Also the uniform asymmetry can be shown in the system response of the overdamped system, after the states values parameters of the asymmetric dichotomous noise are adjusted properly. Then we obtain the averaged power spectrum by the Fourier transform of the autocorrelation function. It is observed that there are three obvious peaks in the averaged powering spectrum. And when the damping coefficient is increased gradually from the underdamping to overdamping, the three peaks generate various transformations. The two parts above reveal SR phenomena in bistable system with asymmetric dichotomous noise.

Finally, the SNR versus the damping coefficient is researched. It is found that several peaks appear in the SNR under some circumstances. The gradual augment of the amplitude of the periodical signal can make those peaks increase and the position toward the right. The decrease of the forcing frequency of the periodical signal can induce that the two peaks of the SNR rise and then decline and 
one peak disappears in the end; meanwhile the position of the peaks move toward the right. And one peak of the SNR develops to two and three peaks with the decrease of the states of the asymmetric and symmetric dichotomous noise. Also the reduction of the noise intensity of the asymmetric dichotomous noise can arouse two or three peaks of the SNR. Furthermore, the states and the intensity of the asymmetric dichotomous noise do not make the position of the peaks of the SNR move toward left and right. And this various situations of the peaks of the SNR versus the damping coefficient demonstrate the existence of SMR phenomena in the bistable system with asymmetric dichotomous noise.

\section{Conflict of Interests}

The authors declare that there is no conflict of interests regarding the publication of this paper.

\section{Acknowledgments}

This work was supported by the NSF of China (Grant nos. 11372247, 11102157, and 11401012), the Research Foundation of Beifang University of Nationalities (Grant nos. 2014XYZ11, 2014XYZ05), and the Research Foundation of School of Mathematics \& Information Science (Grant nos. 13XYK03).

\section{References}

[1] R. Benzi, A. Sutera, and A. Vulpiani, "The mechanism of stochastic resonance," Journal of Physics A, vol. 14, no. 11, pp. L453L457, 1981.

[2] L. Gammaitoni, P. Hänggi, P. Jung, and F. Marchesoni, "Stochastic resonance," Reviews of Modern Physics, vol. 70, no. 1, pp. 223287, 1998.

[3] J. M. G. Vilar and J. M. Rubí, "Stochastic multiresonance," Physical Review Letters, vol. 78, no. 15, pp. 2882-2885, 1997.

[4] J. M. G. Vilar and J. M. Rubí, "Scaling concepts in periodically modulated noisy systems," Physica A: Statistical Mechanics and its Applications, vol. 264, no. 1-2, pp. 1-14, 1999.

[5] A. S. Pikovsky and J. Kurths, "Coherence resonance in a noisedriven excitable system," Physical Review Letters, vol. 78, no. 5, pp. 775-778, 1997.

[6] A. Zaikin, K. Murali, and J. Kurths, "Simple electronic circuit model for doubly stochastic resonance," Physical Review E, vol. 63, Article ID 020103, 2001.

[7] I. Goychuk and P. Hänggi, "Quantum stochastic resonance in symmetric systems," Physical Review E: Statistical Physics, Plasmas, Fluids, and Related Interdisciplinary Topics, vol. 59, no. 5, pp. 5137-5141, 1999.

[8] A. Zaikin, J. Kurths, and L. Schimansky-Geier, "Doubly Stochastic Resonance," Physical Review Letters, vol. 85, no. 2, pp. 227-231, 2000.

[9] R. Rozenfeld, J. A. Freund, A. Neiman, and L. SchimanskyGeier, "Noise-induced phase synchronization enhanced by dichotomic noise," Physical Review E: Statistical, Nonlinear, and Soft Matter Physics, vol. 64, no. 5, Article ID 051107, 2001.

[10] C. Palenzuela, R. Toral, C. R. Mirasso, O. Calvo, and J. D. Gunton, "Coherence resonance in chaotic systems," Europhysics Letters, vol. 56, no. 3, pp. 347-353, 2001.

[11] J. F. Lindner, J. Mason, J. Neff, B. J. Breen, W. L. Ditto, and A. R. Bulsara, "Noninvasive control of stochastic resonance," Physical
Review E: Statistical, Nonlinear, and Soft Matter Physics, vol. 63, no. 4 I, pp. 411071-411078, 2001.

[12] K. Park, Y.-C. Lai, Z. Liu, and A. Nachman, "Aperiodic stochastic resonance and phase synchronization," Physics Letters A, vol. 326, no. 5-6, pp. 391-396, 2004.

[13] L. Gammaitoni, M. Löcher, A. Bulsara et al., "Controlling stochastic resonance," Physical Review Letters, vol. 82, no. 23, pp. 4574-4577, 1999.

[14] G. Jeon and M. Choi, "Autonomous stochastic resonance in fully frustrated Josephson-junction ladders," Physical Review B, vol. 66, Article ID 064514, 2002.

[15] Y. Xu, X. Jin, H. Zhang, and T. Yang, "The availability of logical operation induced by dichotomous noise for a nonlinear bistable system," Journal of Statistical Physics, vol. 152, no. 4, pp. 753-768, 2013.

[16] Y. Xu, X. Jin, and H. Zhang, "Parallel logic gates in synthetic gene networks induced by non-Gaussian noise," Physical Review E, vol. 88, no. 5, Article ID 052721, 2013.

[17] R. Ray and S. Sengupta, "Stochastic resonance in underdamped, bistable systems," Physics Letters A, vol. 353, pp. 364-371, 2006.

[18] Y. Xu, J. Li, J. Feng, H. Zhang, W. Xu, and J. Duan, "Lévy noiseinduced stochastic resonance in a bistable system," The European Physical Journal B, vol. 86, p. 198, 2013.

[19] L. Zhang, S. Zhong, H. Peng, and M. Luo, "Stochastic multiresonance in a linear system driven by multiplicative polynomial dichotomous noise," Chinese Physics Letters, vol. 28, no. 9, Article ID 090505, 2011.

[20] L. Gammaitoni, E. Menichella-Saetta, S. Santucci, F. Marchesoni, and C. Presilla, "Periodically time-modulated bistable systems: stochastic resonance," Physical Review A, vol. 40, no. 4, pp. 2114-2119, 1989.

[21] L. Gammaitoni, F. Marchesoni, E. Menichella-Saetta, and S. Santucci, "Stochastic resonance in bistable systems," Physical Review Letters, vol. 62, no. 4, pp. 349-352, 1989.

[22] L. Gammaitoni and A. R. Bulsara, "Stochastic resonance in underdamped, bistable systems," in The Model, J. Freund and T. Poschel, Eds., vol. 557 of LNP, p. 131, 2000.

[23] F. Guo, Y.-R. Zhou, and Y. Zhang, "Stochastic resonance in a time-delayed bistable system subjected to dichotomous noise and white noise," Chinese Journal of Physics, vol. 48, no. 2, pp. 294-303, 2010

[24] J. Li and Y. Han, "Stochastic resonance induced by Dichotomous resistor in an electric circuit," Communications in Theoretical Physics, vol. 47, p. 487, 2007.

[25] Y. Jin, W. Xu, M. Xu, and T. Fang, "Stochastic resonance in linear system due to dichotomous noise modulated by bias signal," Journal of Physics A: Mathematical and General, vol. 38, no. 17, pp. 3733-3742, 2005.

[26] J. Li and Y. Han, "Phenomenon of stochastic resonance caused by multiplicative asymmetric dichotomous noise," Physical Review E, vol. 74, no. 5, Article ID 051115, 9 pages, 2006.

[27] F. Guo and Y.-R. Zhou, "Stochastic resonance in a stochastic bistable system subject to additive white noise and dichotomous noise," Physica A, vol. 388, no. 17, pp. 3371-3376, 2009.

[28] D. Barik, P. K. Ghosh, and D. S. Ray, "Langevin dynamics with dichotomous noise; direct simulation and applications," Journal of Statistical Mechanics, vol. 2006, p. P03010, 2006.

[29] S. Mitain and B. Kosko, "Adaptive stochastic resonance," Proceedings of the IEEE, vol. 86, no. 11, pp. 2152-2183, 1998. 


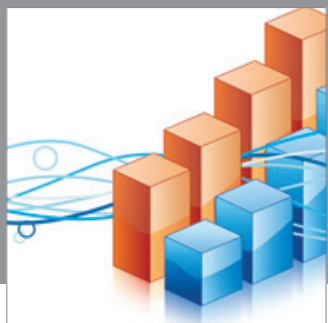

Advances in

Operations Research

mansans

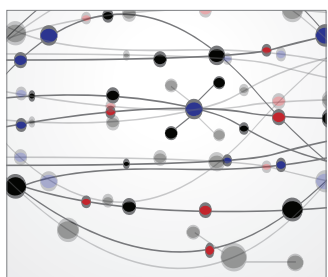

The Scientific World Journal
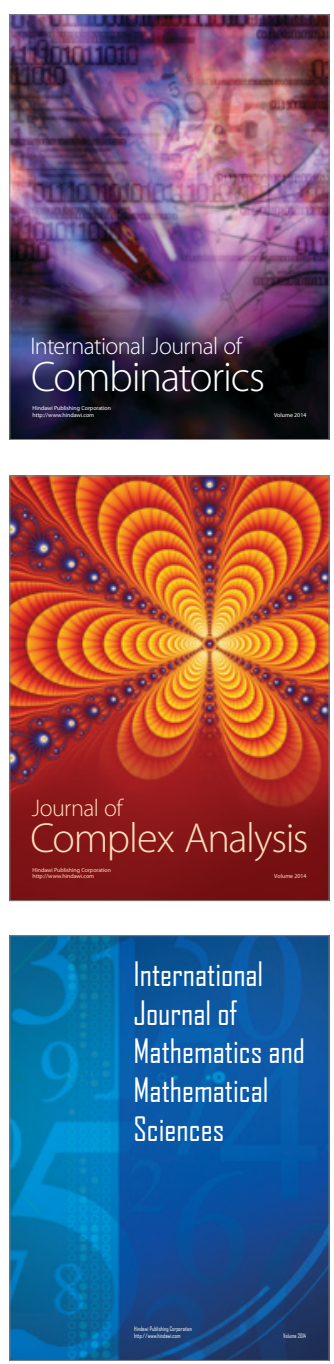
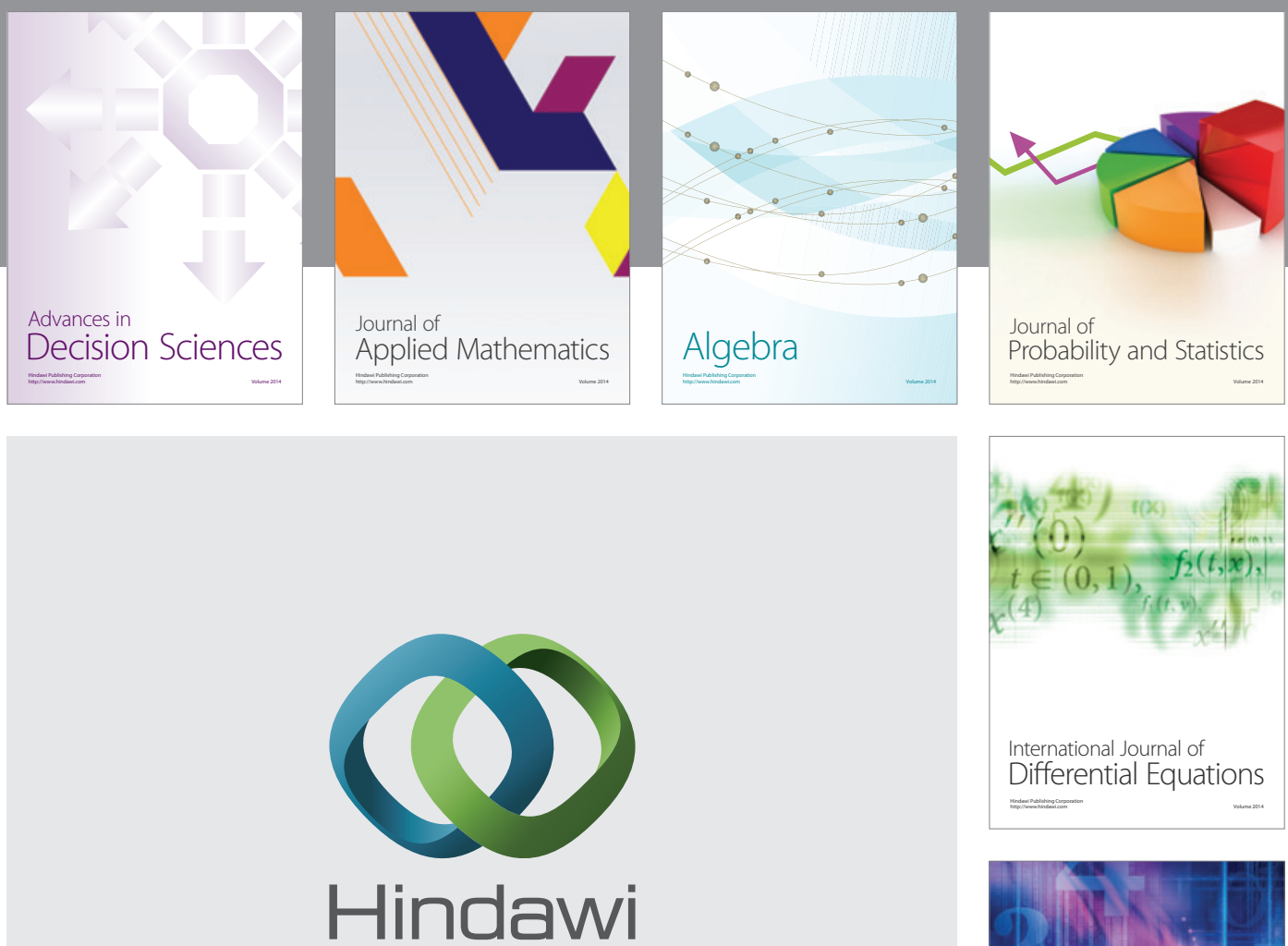

Submit your manuscripts at http://www.hindawi.com
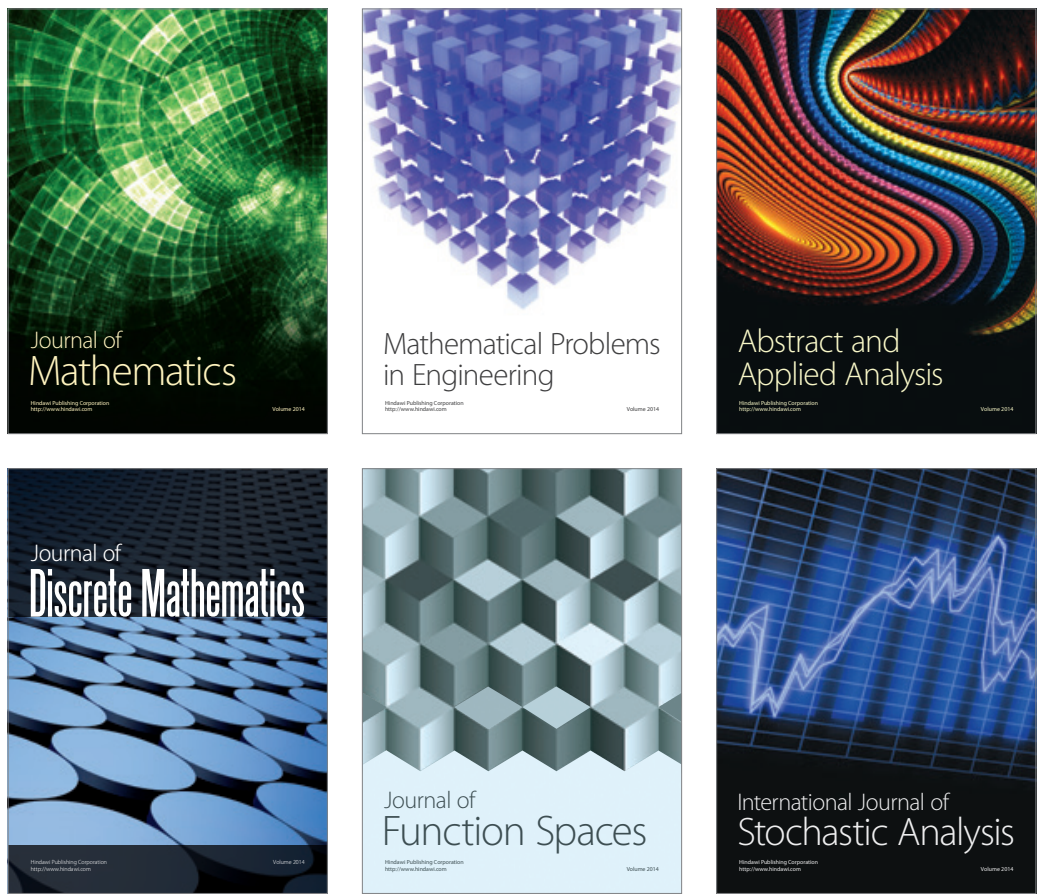

Journal of

Function Spaces

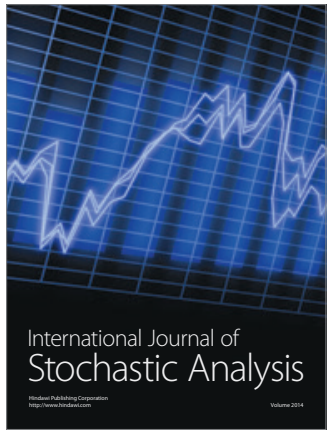

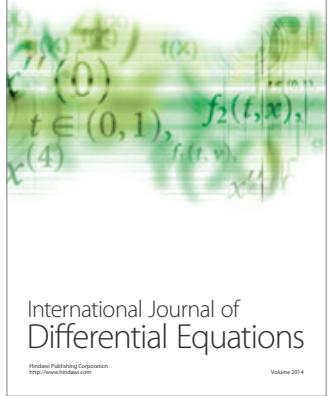
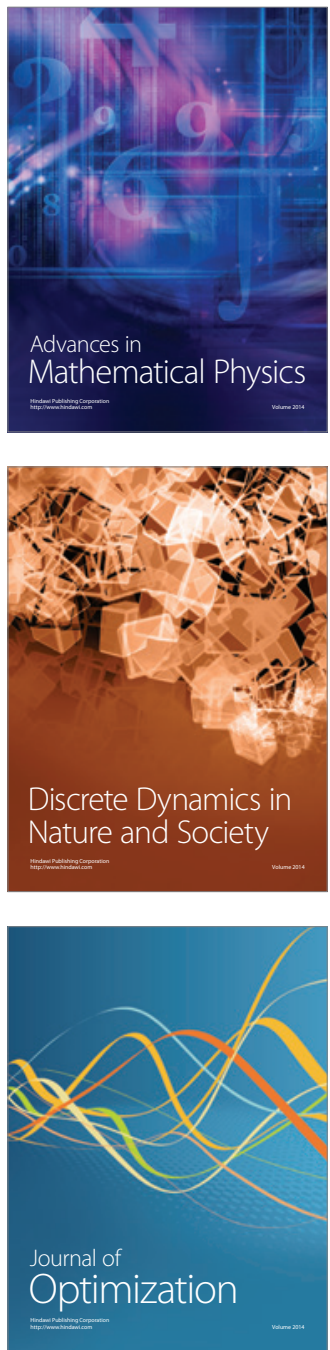